\title{
Powerful nonparametric seasonal unit root tests
}

\author{
Burak Alparslan Eroğlu ${ }^{\mathrm{a}, *}$, Kemal Çağlar Göğebakan ${ }^{\mathrm{b}}$, Mirza Trokićc,1 \\ a Department of Economics, Istanbul Bilgi University, Istanbul, Turkey \\ ${ }^{\mathrm{b}}$ Department of Economics, Bilkent University, Ankara, Turkey \\ c IHS Markit, Eviews, 4199 Campus Dr Irvine, CA 92612, USA
}

\section{H I G H L I G H T S}

- A family of nonparametric seasonal unit root tests is proposed.

- The proposed test statistics do not require estimation of any regression model.

- The proposed tests use fractional integration techniques.

- In the Monte Carlo simulations, our tests exhibit satisfactory power properties.

\section{A R T I C L E I N F O}

\section{Article history:}

Received 18 February 2018

Received in revised form 10 March 2018

Accepted 14 March 2018

Available online 23 March 2018

\section{JEL classification:}

C22

C12

C14

Keywords:

Seasonal unit roots

Fractional integration

Non-parametric

\begin{abstract}
A B S T R A C T
This paper introduces a powerful nonparametric testing procedure for seasonal unit roots by utilizing the fractional integration operator. Different from the well-known seasonal unit root tests of Hylleberg et al. (1990), the proposed tests do not require any parametric specifications.
\end{abstract}

(C) 2018 Elsevier B.V. All rights reserved.

\section{Introduction}

After the seminal work of Hylleberg et al. (1990) [HEGY], seasonal unit root testing has attracted attention in theoretical and empirical studies. The HEGY tests are the seasonal generalization of the augmented Dickey-Fuller [ADF] unit root test and allow researchers to test for unit root behavior at each of the zero, Nyquist and seasonal frequencies. Recently, HEGY's framework has been extended and improved by many subsequent authors

\footnotetext{
* Correspondence to: Eski Silahtarağa Elektrik Santralı Kazım Karabekir Cad., No: 2/13 34060 Eyüpsultan İstanbul, Turkey.

E-mail addresses: burak.eroglu@bilgi.edu.tr (B.A. Eroğlu), caglar.gogebakan@bilkent.edu.tr (K.Ç. Göğebakan), mirza.trokic@ihsmarkit.com (M. Trokić).

1 Opinions, statements, estimates and projections in this article are solely those of the individual author(s). They do not necessarily reflect the opinions of IHS Markit or any of its affiliates ("IHS Markit"). IHS Markit has no obligation to update, modify or amend this article, or to otherwise notify readers, in the event that any matter stated herein, or any opinion, projection, forecast or estimate set forth herein, changes or subsequently becomes inaccurate.
}

such as Rodrigues and Taylor (2007), Smith et al. (2009) and Barrio Castro et al. (2012) [BOT]. Despite these improvements, these tests still use parametric lag augmentation to account for the serial correlation. Therefore, their size and power performance highly depend on the selection of the lag length parameter in the augmented regression. Motivated by these issues, Taylor (2005) is the only serious attempt to construct non-parametric seasonal variance ratio unit root tests. In this regard, we aim to propose a new family of non-parametric seasonal unit root tests, which are free from parametric lag augmentation and improve the existing non-parametric seasonal unit root tests in terms of asymptotic local power.

This new testing design is an extension of Nielsen's (2009) nonparametric variance ratio test to the seasonal unit root context. Consequently, the proposed seasonal non-parametric unit root tests use the fractional integration and the family of these tests is indexed by the fractional integration parameter $d$. The fractional integration techniques have received a large amount of attention in the economics literature (see Robinson (2003)). We show that 
these techniques are useful to construct powerful unit root tests at the zero, Nyquist and seasonal frequencies.

To improve the power of the parametric seasonal unit root tests, Gregoir (2006) and Rodrigues and Taylor (2007) propose feasible tests that are nearly efficient (GLS de-trended) in the seasonal context. More recently, Jansson and Nielsen (2011) develop nearly efficient likelihood ratio tests for the seasonal unit root hypothesis. Accordingly, we also present GLS de-trended versions of our nonparametric seasonal unit root tests. Besides these, we also propose non-parametric joint unit root tests for the seasonal frequencies. With this contribution, we extend the scope of the non-parametric seasonal unit root testing framework. The proofs and simulation results are included in the Appendix.

\section{The seasonal unit root model}

We consider the univariate seasonal time series $\left\{x_{S t+s}\right\}$ with the following data generating process (DGP) ${ }^{2}$ :

$\alpha(L) x_{S t+s}=u_{S t+s}, \quad s=1-S, \ldots, 0, \quad t=1,2, \ldots, N$

$u_{S t+s}=\psi(L) \varepsilon_{S t+s}$

where $S$ is the number of seasons, $\alpha(L)=1-\sum_{j=1}^{S} \alpha_{j} L^{j}$ is an $S$ order lag polynomial which determines the seasonal unit root. Further, the serial correlation structure is governed by the lag polynomial $\psi(L)=1+\sum_{j=1}^{\infty} \psi_{j} L^{j}$. The total sample size is given by $T=S N$, where $N$ denotes the total number of observations in each season. We assume that the initial conditions $x_{1-S}, \ldots, x_{0}$ are $o_{p}\left(T^{1 / 2}\right)$. Assumptions on the innovation sequence $\left\{\varepsilon_{S t+s}\right\}$ and on the lag polynomial $\psi(L)$ are given as:

Assumption A.1. Let $\left(\varepsilon_{S t+s}, \mathcal{F}_{S t+s}\right)$ be a martingale difference sequence, with filtration $\left(\mathcal{F}_{S t+s}\right)$, where $\mathcal{F}_{S t+s} \subset \mathcal{F}_{S t+s+1}$ for all $s, t$ such that (a) $\mathbb{E}\left(\varepsilon_{S t+s}^{2} \mid \mathcal{F}_{S t+s-1}\right)=\sigma^{2}$ (b) $\frac{1}{N} \sum_{t=1}^{N} \varepsilon_{S t+s}^{2} \stackrel{p}{\longrightarrow} \sigma^{2}$ for all $s=1-S, \ldots, 0$ (c) $\mathbb{E}\left|\varepsilon_{S t+s}\right|^{r}<K<\infty$ for some $r \geq 4$.

Assumption A.2. The power series $\psi(z) \neq 0$ for all $|z| \leq 1$, and $\sum_{j=0}^{\infty} h^{h}\left|\psi_{j}\right|<\infty$ for some $h \geq 1$.

These two assumptions are standard in seasonal unit root literature. The detailed discussion about the given assumptions can be found in BOT.

In this study, we focus on testing whether $x_{S t+s}$ in (1) is a seasonally integrated process. The corresponding null hypothesis is given as:

$H_{0}: \alpha(L)=\left(1-L^{S}\right)=: \Delta_{S}$.

In this hypothesis, the $A R(S)$ polynomial $\alpha(L)$ can be factorized as $\alpha(L)=\prod_{j=0}^{\lfloor S / 2\rfloor} \omega_{j}(L)$ where $\omega_{0}(L)=\left(1-\alpha_{0} L\right)$ corresponds to the root at the zero frequency $\omega_{0}:=0$ with the parameter $\alpha_{0}, \quad \omega_{j}(L)=\left[1-2\left(\alpha_{j} \cos \omega_{j}-\beta_{j} \sin \omega_{j}\right) L+\left(\alpha_{j}^{2}+\beta_{j}^{2}\right) L^{2}\right]$ associates the parameters $\alpha_{j}$ and $\beta_{j}, \quad j=1, \ldots, S^{*}, S^{*}=\lfloor(S-1) / 2\rfloor$ with the harmonic seasonal frequencies $\left(\omega_{j}, 2 \pi-\omega_{j}\right), \quad \omega_{j}=2 \pi j / S$, and for $S$ is even, $\omega_{S / 2}(L):=\left(1+\alpha_{S / 2} L\right)$ corresponds to the Nyquist frequency $\omega_{S / 2}:=\pi$ with the parameter $\alpha_{S / 2}$ (for the details see BOT).

Therefore, the null hypothesis can be partitioned as $H_{0}=$ $\cap_{j=0}^{\lfloor S / 2\rfloor} H_{0, j}$, where

$H_{0, i}: \alpha_{i}=1, \quad i=0, S / 2$

$H_{0, j}: \alpha_{j}=1$ and $\beta_{j}=0 \quad j=1, \ldots, S^{*}$.

2 The notation and assumptions closely follow BOT.
The alternative hypothesis is $H_{1}=\cup_{j=0}^{\lfloor S / 2\rfloor} H_{1, j}$ which ensures stationarity at one or more of the zero frequency or seasonal frequencies, where

$H_{1, i}:\left|\alpha_{i}\right|<1, \quad i=0, S / 2$

$H_{1, j}: \alpha_{j}^{2}+\beta_{j}^{2}<1 \quad j=1, \ldots, S^{*}$.

\section{Fractional seasonal variance ratio unit root tests (FSVR)}

To construct our nonparametric FSVR unit root tests, we first define the $S$ dimensional process $X_{t}$ which separates the observed series $x_{S t+s}$ from (1) into sub-seasonal observations:

$X_{t}:=\left[x_{S t-(S-1)}, x_{S t-(S-2)}, \ldots, x_{S t}\right]^{\prime} \quad t=0, \ldots, N$.

In our tests, we utilize the fractional integration operator. This operator is defined by:

$$
\begin{aligned}
\tilde{y}_{t} & :=\Delta_{+}^{-d} y_{t}=(1-L)_{+}^{-d} y_{t}=\sum_{k=0}^{t-1} \frac{\Gamma(k+d)}{\Gamma(d) \Gamma(k+1)} y_{t-k} \\
& =\sum_{k=0}^{t-1} \pi_{k}(d) y_{t-k}
\end{aligned}
$$

where $\Gamma(\cdot)$ is gamma function and $\pi_{k}(d)$ is the fractional binomial coefficient. The integration order satisfies $d>0$ and it is selected by the practitioner. We apply this operator to each element of $X_{t}$ separately to obtain:

$\tilde{X}_{t}=\Delta_{+}^{-d} X_{t}=\left[\Delta_{+}^{-d} x_{S t-(S-1)}, \Delta_{+}^{-d} x_{S t-(S-2)}, \ldots, \Delta_{+}^{-d} x_{S t}\right]^{\prime}$ for all

$t=1, \ldots, N$.

After obtaining $\tilde{X}_{t}$, we create a new variable, say $\tilde{x}_{S t+s}$ from $\tilde{X}_{t}$ as each vector of $\tilde{X}_{t}$ corresponds to one season of the final process $\tilde{x}_{S t+s}$. This process is very different than the fractional integration of $x_{S t+s}$ which is written as $\Delta_{+}^{-d} x_{S t+s}, s=1-S, \ldots 0, t=1, \ldots, N$.

To define our test statistics, first we need to apply the HEGY transformations on the observed series $x_{S t+s}$ and the transformed series $\tilde{x}_{S t+s}: x_{0, S t+s}$ is given in Box I. Using these objects, we define the test statistics for testing unit root hypothesis at different frequencies. First, consider the test statistic of unit root at the zero and Nyquist frequencies:

$\tau_{k}(d)=N^{2 d} \frac{\sum_{t=1}^{N} \sum_{s=1-S}^{0} x_{k, S t+s}^{2}}{\sum_{t=1}^{N} \sum_{S=1-S}^{0} \tilde{x}_{k, S t+s}^{2}}$ for $k=0, S / 2$.

For the harmonic frequencies, we propose two variance ratio test statistics:

$\tau_{j}(d)=N^{2 d} \frac{\sum_{t=1}^{N} \sum_{s=1-S}^{0} x_{j, S t+s}^{2}}{\sum_{t=1}^{N} \sum_{s=1-S}^{0} \tilde{x}_{j, S t+s}^{2}}$ for $j=1, \ldots, S^{*}$

$\tau_{j}^{*}(d)=N^{2 d} \frac{\sum_{t=1}^{N} \sum_{s=1-S}^{0} x_{j, S t+s}^{* 2}}{\sum_{t=1}^{N} \sum_{s=1-S}^{0} \tilde{x}_{j, S t+s}^{* 2}}$ for $j=1, \ldots, S^{*}$

where $\tau_{j}(d)$ and $\tau_{j}^{*}(d)$ are the test statistics for the unit root hypothesis at the harmonic frequencies $\omega_{j}$ and $2 \pi-\omega_{j}$ for all $j=$ $1, \ldots, S^{*}$, respectively. Furthermore, the following test statistic is for testing the presence of the pairs of complex conjugate unit roots:

$\tilde{\tau}_{j}(d)=\left(\tau_{j}(d)+\tau_{j}^{*}(d)\right) / 2$ for $j=1, \ldots, S^{*}$.

Finally, we derive the $F$ type statistics for the joint test for the unit roots in the different frequencies:

$\tau_{1, \ldots,\lfloor S / 2\rfloor}(d)=\frac{1}{S-1}\left(\tau_{S / 2}(d)+\sum_{j=1}^{S^{*}} \tau_{j}(d)+\sum_{j=1}^{S^{*}} \tau_{j}^{*}(d)\right)$ 


$$
\begin{aligned}
x_{0, S t+s} & :=\sum_{j=0}^{S-1} x_{S t+s-j} & \tilde{x}_{0, S t+s}:=\sum_{j=0}^{S-1} \tilde{x}_{S t+s-j} \\
x_{S / 2, S t+s}:= & \sum_{j=0}^{S-1} \cos [(j+1) \pi] x_{S t+s-j} & \tilde{x}_{S / 2, S t+s}:=\sum_{j=0}^{S-1} \cos [(j+1) \pi] \tilde{x}_{S t+s-j} \\
x_{i, S t+s}:= & \sum_{j=0}^{S-1} \cos \left[(j+1) \omega_{i}\right] x_{S t+s-j} \text { for all } i=1, \ldots, S^{*} & \tilde{x}_{i, S t+s}:=\sum_{j=0}^{S-1} \cos \left[(j+1) \omega_{i}\right] \tilde{x}_{S t+s-j} \text { for all } i=1, \ldots, S^{*} \\
x_{i, S t+s}^{*}:= & \sum_{j=0}^{S-1} \sin \left[(j+1) \omega_{i}\right] x_{S t+s-j} \text { for all } i=1, \ldots, S^{*} & \tilde{x}_{i, S t+s}^{*}:=\sum_{j=0} \sin \left[(j+1) \omega_{i}\right] \tilde{x}_{S t+s-j} \text { for all } i=1, \ldots, S^{*} .
\end{aligned}
$$

\section{Box I.}

$\tau_{0, \ldots,\lfloor S / 2\rfloor}(d)=\frac{1}{S}\left(\tau_{0}(d)+\tau_{S / 2}(d)+\sum_{j=1}^{S^{*}} \tau_{j}(d)+\sum_{j=1}^{S^{*}} \tau_{j}^{*}(d)\right)$.

Remark 1. The test statistics in (8) and (9) correspond to the joint unit root tests at all frequencies except the zero frequency and the joint unit root tests at all frequencies, respectively.

\section{Asymptotic analysis}

To derive the asymptotic results of the FSVR tests, under the overall null hypothesis, we write $\left\{x_{S t+s}\right\}$ in $S$ dimensional vector form:

$X_{t}=X_{t-1}+U_{t}$

$X_{t}:=\left[x_{S t-(S-1)}, x_{S t-(S-2)}, \ldots, x_{S t}\right]^{\prime} \quad t=0, \ldots, N$

$U_{t}:=\left[u_{S t-(S-1)}, u_{S t-(S-2)}, \ldots, u_{S t}\right]^{\prime} \quad t=0, \ldots, N$

where the innovation process $U_{t}$ can be written as:

$U_{t}=\sum_{j=0}^{\infty} \Psi_{j} E_{t-j}$

$E_{t}:=\left[\varepsilon_{S t-(S-1)}, \varepsilon_{S t-(S-2)}, \ldots, \varepsilon_{S t}\right]^{\prime} \quad t=0, \ldots, N$

where $\Psi_{j}, j=0,1, \ldots$ are defined in BOT. Consider the following lemma:

Lemma 1. Let $X_{t}$ is generated as in Eqs. (10). Then under Assumptions A.1-A.2,

$N^{-1 / 2} X_{\lfloor r N\rfloor} \Rightarrow \sigma \Psi(1) W(r) \quad r \in[0,1]$

where $W(r)$ is $S$ dimensional standard Brownian motion process.

The following lemma demonstrates the limiting distribution of the fractionally transformed process:

Lemma 2. Let $X_{t}$ is generated as in Eq. (10) and $\tilde{X}_{t}$ is obtained from Eq. (3). Under Assumptions A.1-A.2, then

$N^{d-1 / 2} \tilde{X}_{\lfloor r N\rfloor} \Rightarrow \sigma \Psi(1) W_{d+1}(r) \quad r \in[0,1]$

where

$W_{d+1}(r)=\frac{1}{\Gamma(d+1)} \int_{0}^{r}(r-t)^{d} d W(t)$

is $S$ dimensional type II Fractional Brownian motion.

Using Lemmas 1 and 2, we obtain the following asymptotic results for the FSVR test statistics:
Theorem 1. Let the assumptions of Lemma 1 hold. Consider the FSVR test statistics given by Eqs. (4)-(9). As $N \longrightarrow \infty$,

$$
\begin{aligned}
& \tau_{i}(d) \Rightarrow \frac{\int_{0}^{1} W_{i}(r)^{2} d r}{\int_{0}^{1} W_{d+1, i}(r)^{2} d r}=\eta_{i}(d) \quad i=0, S / 2 \\
& \tau_{j}(d) \Rightarrow \frac{\int_{0}^{1} W_{j}(r)^{2} d r+\int_{0}^{1} W_{j}^{*}(r)^{2} d r}{\int_{0}^{1} W_{d+1, j}(r)^{2} d r+\int_{0}^{1} W_{d+1, j}^{*}(r)^{2} d r}=\eta_{j}(d) \text { for } \\
& j=1, \ldots, S^{*} \\
& \tau_{j}^{*}(d) \Rightarrow \eta_{j}(d) \text { for } j=1, \ldots, S^{*} \\
& \tilde{\tau}_{j}(d) \Rightarrow \eta_{j}(d) \text { for } j=1, \ldots, S^{*} \\
& \tau_{1, \ldots,\lfloor S / 2\rfloor}(d) \Rightarrow \frac{1}{S-1}\left(\eta_{S / 2}(d)+2 \sum_{j=1}^{S^{*}} \eta_{j}(d)\right) \\
& \tau_{0, \ldots,\lfloor S / 2\rfloor}(d) \Rightarrow \frac{1}{S}\left(\eta_{0}(d)+\eta_{S / 2}(d)+2 \sum_{j=1}^{S^{*}} \eta_{j}(d)\right)
\end{aligned}
$$

where $W_{0}(r), W_{S / 2}(r), W_{j}^{*}(r)$ and $W_{j}(r)$ for $j=1, \ldots, S^{*}$ are the independent standard Brownian motions, $W_{d+1,0}(r), W_{d+1, S / 2}(r)$, $W_{d+1, j}^{*}(r)$ and $W_{d+1, j}(r)$ are the independent fractional Brownian motions for $j=1, \ldots, S^{*}$, respectively.

Remark 2. In Theorem 1, to adjust for the deterministic elements, we extend these results by the following generalization:

$y_{S t+s}=\gamma^{\prime} z_{S t+s}+x_{S t+s}, s=1-S, \ldots, 0$ and $t=1, \ldots, N$

where $x_{S t+s}$ is defined in (1)-(2) and $Z_{S t+s}$ are purely deterministic. Following Smith et al. (2009), we define the characterization of $\mu_{S t+s}$ with a typology of three cases, namely: no deterministic component $(j=0)$, seasonal intercepts $(j=1)$, seasonal intercepts and seasonal trends $(j=2)$. Our test statistics (4)-(9) can be redefined using OLS de-trended series, say $\hat{x}_{S t+s}=y_{S t+s}-\hat{\gamma}_{O L S}^{\prime} z_{S t+s}$, as in Smith et al. (2009). Accordingly, the Brownian motions in the limiting distributions are replaced by the OLS demeaned or de-trended analogues of the Brownian motions and type II fractional Brownian motions. Next, instead of the simple OLS detrending, following Rodrigues and Taylor (2007), we obtain the demeaned or de-trended series by using the GLS-detrending algorithm. Let $Y_{t}:=\left[y_{S t-(S-1)}, y_{S t-(S-2)}, \ldots, y_{S t}\right]^{\prime}$ be defined similarly as in (10). We apply quasi-differencing to $Y_{t}$ and $Z_{t}$ where $Z_{t}:=$ 
$\left[z_{S t-(S-1)}, z_{S t-(S-2)}, \ldots, z_{S t}\right]^{\prime}:$

$Y_{\bar{c}}=\left[Y_{1}, Y_{2}-\left(1-\frac{\bar{c}}{N}\right) Y_{1}, \ldots, Y_{N}-\left(1-\frac{\bar{c}}{N}\right) Y_{N-1}\right]^{\prime}$

$Z_{\bar{c}}=\left[Z_{1}, Z_{2}-\left(1-\frac{\bar{c}}{N}\right) Z_{1}, \ldots, Z_{N}-\left(1-\frac{\bar{c}}{N}\right) Z_{N-1}\right]^{\prime}$.

We can obtain the coefficient $\hat{\gamma}_{G L S}$ by regressing $Y_{\bar{c}}$ on $Z_{\bar{c}}$. Then, the GLS de-trended series are given by converting the vector process $\hat{X}_{t}=Y_{t}-\hat{\gamma}_{G L S}^{\prime} Z_{t}$ to the seasonal process $\hat{x}_{S t+s}$ where $\hat{X}_{t}:=$ $\left[\hat{x}_{S t-(S-1)}, \hat{x}_{S t-(S-2)}, \ldots, \hat{x}_{S t}\right]^{\prime}$. We replace these GLS de-trended series with $x_{S t+s}$ in the testing procedure. Finally, the asymptotic distributions are replaced with the GLS demeaned or de-trended versions of Brownian Motions and Fractional Brownian motions. These objects are same as described in Nielsen (2009).

Remark 3. Let $C V_{\gamma, S}\left(\eta_{j}(d)\right)$ be the $\gamma$ th quantile of the asymptotic distribution of the test statistic $\tau_{j}(d)$ for $j=0, \ldots, S / 2$. We reject the one sided $t$ type test if $\tau_{j}(d)<C V_{\gamma, S}\left(\eta_{j}(d)\right)$ for all $j=0, \ldots, S / 2$. For the joint tests, we reject the null hypothesis if $\tau_{0, \ldots,\lfloor S / 2\rfloor}(d)>C V_{(1-\gamma), S}\left(\eta_{0, \ldots,\lfloor S / 2\rfloor}(d)\right)$ where $C V_{(1-\gamma), S}\left(\eta_{0, \ldots,\lfloor S / 2\rfloor}(d)\right)$ is the $(1-\gamma)$ th quantile of the asymptotic distribution of $\tau_{0, \ldots,\lfloor S / 2\rfloor}(d)$.

Remark 4. For $d=1$, Taylor's (2005) tests and inverse of our tests are equivalent.

\section{Finite sample simulations}

In the Monte Carlo simulations, data is generated according to the quarterly $(S=4)$ DGP as follows:

$$
\begin{aligned}
& x_{4 t+s}=(1-c / N) x_{4 t+s-1}+u_{4 t+s}, \quad s=-3, \ldots, 0, \\
& \quad t=1,2, \ldots, N \\
& \phi(L) u_{4 t+s}=\theta(L) \varepsilon_{4 t+s}, \varepsilon_{4 t+s} \sim \operatorname{NIID}(0,1)
\end{aligned}
$$

with $\varepsilon_{4 t+s}=0$ for $t \leq 0$ and $c$ is the local-to-unity parameter. We pick $c=0$ for the size and $c \in\{7,13.5\}$ for the size adjusted power evaluation. All simulations are conducted 10,000 times with $N=100$ and 0.05 nominal significance level with demeaned data. The critical values are obtained by simulation and available upon request.

We consider 5 scenarios for the serial correlation structure in the innovations. First one does not contain any serial correlation. For the other scenarios, $u_{4 t+s}$ follows $\operatorname{ARMA}(1,1)$ process: $(1-$ $0.5 L) u_{4 t+s}=(1-0.5 L) \varepsilon_{4 t+s}, \operatorname{ARMA}(2,2)$ process: $\left(1+0.5 L^{2}\right) u_{4 t+s}=$ $\left(1+0.5 L^{2}\right) \varepsilon_{4 t+s}, M A(4)$ process: $u_{4 t+s}=\left(1+0.5 L^{4}\right) \varepsilon_{4 t+s}$ and ARMA $(4,4)$ process: $\left(1-0.5 L^{4}\right) u_{4 t+s}=\left(1-0.5 L^{4}\right) \varepsilon_{4 t+s}$.

The finite sample FSVR test results are reported for the test statistics $\tau_{0}(d), \tau_{S / 2}(d), \tilde{\tau}_{1}(d), \tau_{0, \ldots,\lfloor S / 2\rfloor}(d)$ and $\tau_{1, \ldots,\lfloor S / 2\rfloor}(d)$, and the performance is compared with the HEGY counterparts $t_{0}, t_{\lfloor S / 2\rfloor}, F_{1}$, $F_{0, \ldots,\lfloor S / 2\rfloor}$ and $F_{1, \ldots,\lfloor S / 2\rfloor}$ in Table 1 . The table contains the results with both OLS and GLS demeaning. We set $\bar{c}=13$. . $^{3}$

Following Nielsen (2009), we investigate the impact of the fractional integration parameter $d$ in the performance of FSVR tests. The best results in terms of power and size are obtained with $d=0.1$. For comparison with Taylor's (2005) tests, we also report the case with $d=1{ }^{4}$ The results indicate that the FSVR and HEGY tests are correctly sized in the OLS and GLS demeaning cases. For $d=0.1$, the FSVR tests perform better than the HEGY tests when OLS demeaning is used. The GLS demeaning improves

\footnotetext{
3 For HEGY, the optimal lag length selection routine of Barrio Castro et al. (2016) is used.

4 The results with other values of $d$ and $\bar{c}$ are available upon request.
}

the power performance of the FSVR procedure compared to the OLS demeaning. In the GLS demeaning case, HEGY tests have advantage in the individual coefficient testing relative to the FSVR tests. However, in this case, the FSVR and HEGY methods enjoy similar power properties in the joint tests.

\section{Conclusion}

In this paper, we construct a family of powerful non-parametric seasonal unit root tests at the zero, Nyquist, harmonic and joint frequencies. The simulation results reveal that our proposed nonparametric tests show competitive power performance with the parametric HEGY tests with desirable size properties in all scenarios considered.

\section{Appendix}

\section{A.1. Proofs}

Throughout the proofs, we use the circulant matrices:

$$
\begin{aligned}
& C_{0}=\operatorname{Circ}[1,1,1, \ldots, 1]=v_{0} v_{0}^{\prime} \\
& C_{S / 2}=\operatorname{Circ}[1,-1,1, \ldots,-1,1]=v_{S / 2} v_{S / 2}^{\prime} \\
& C_{i}=\operatorname{Circ}\left[\cos (0), \cos \left(\omega_{i}, \cos \left(2 \omega_{i}\right)\right), \ldots, \cos \left((S-1) \omega_{i}\right)\right]=v_{i} v_{i}^{\prime} \text { for } \\
& \quad i=1, \ldots, S^{*} \\
& C_{i}^{*}=\operatorname{Circ}\left[\sin (0), \sin \left(\omega_{i}, \sin \left(2 \omega_{i}\right)\right), \ldots, \sin \left((S-1) \omega_{i}\right)\right]=v_{i}^{*} v_{i}^{* \prime} \text { for } \\
& \quad i=1, \ldots, S^{*} .
\end{aligned}
$$

Proof of Lemma 1. The proof can be found in Lemma 1 of BOT.

Proof of Lemma 2. The proof follows from the same argument in proof of Lemma 6.(d) of Nielsen (2010). Since the operator $\Delta_{+}^{-d}$ satisfies $\Delta_{+}^{-d} \Delta_{+}^{-d_{2}} Y_{t}=\Delta_{+}^{-d_{2}} \Delta_{+}^{-d} Y_{t}=\Delta_{+}^{-d-d_{2}} Y_{t}$, we can write

$\tilde{X}_{r}=\Delta_{+}^{-d} \Delta_{+}^{-1} E_{r}=\Delta_{+}^{-1} \Delta_{+}^{-d} E_{r}=\sum_{i=1}^{r} \Delta_{+}^{-d} E_{r}$.

Now define $V_{r}=\Delta_{+}^{-d} E_{r}$ which satisfies the conditions of Theorem 1 in Marinucci and Robinson (2000), we obtain:

$N^{1 / 2-d} \sum_{i=1}^{\lfloor r N\rfloor} V_{i} \Rightarrow \sigma \Psi(1) W_{d+1}(r)$

Proof of Theorem 1. Consider numerator of $\tau_{j}(d)$ for $j=0, S / 2$. The convergence results for these objects are given in BOT as:

$$
\begin{aligned}
& T^{-2} \sum_{t=1}^{N} \sum_{S=1-S}^{0} x_{j, S t+s}^{2}=T^{-2} \sum_{t=1}^{N} S X_{t}^{\prime} C_{j} X_{t}+o_{p}(1) \text { for } j=0, S / 2 \\
& \Rightarrow \frac{1}{S} \int_{0}^{1} W(r)^{\prime} \Psi(1)^{\prime} C_{j} \Psi(1) W(r) d r \\
& =\left\{\begin{array}{l}
\frac{\sigma^{2} \psi(1)^{2}}{S} \int_{0}^{1} W(r)^{\prime} C_{0} W(r) d r \text { if } j=0 \\
\frac{\sigma^{2} \psi(-1)^{2}}{S} \int_{0}^{1} W(r)^{\prime} C_{S / 2} W(r) d r \text { if } j=S / 2 .
\end{array}\right.
\end{aligned}
$$

Similarly, for $j=1, \ldots, S^{*}$ BOT shows that:

$$
\begin{aligned}
& T^{-2} \sum_{\substack{t=1 \\
j=1}}^{N} \sum_{S=1-S}^{0} x_{j, S t+s}^{2}=T^{-2} \sum_{t=1}^{N} \frac{S}{2} X_{t}^{\prime} C_{j} X_{t}+o_{p}(1) \text { for } \\
& \quad j=1, \ldots, S^{*}
\end{aligned}
$$


Table 1

Finite sample size and size-adjusted power for the fractional seasonal variance ratio and HEGY tests with demeaning.

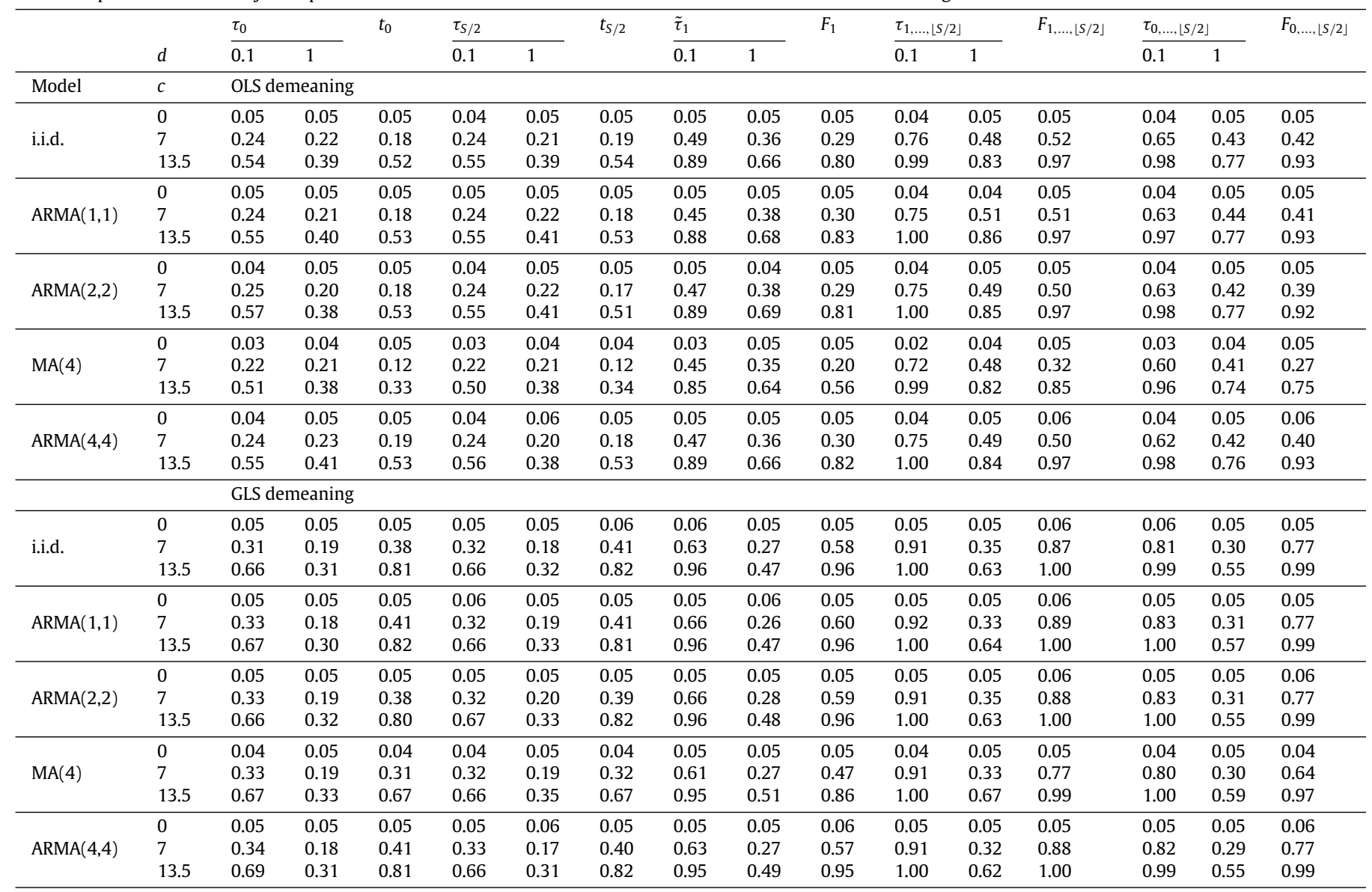

Note: The results for HEGY tests do not depend on $d$.

$$
\begin{aligned}
& \Rightarrow \frac{1}{2 S} \int_{0}^{1} W(r)^{\prime} \Psi(1)^{\prime} C_{j} \Psi(1) W(r) d r \\
& =\frac{\sigma^{2}\left(a_{j}^{2}+b_{j}^{2}\right)}{2 S} \int_{0}^{1} W(r)^{\prime} C_{j} W(r) d r \text { if } j=1, \ldots, S^{*}
\end{aligned}
$$

and

$$
\begin{aligned}
& T^{-2} \sum_{t=1}^{N} \sum_{s=1-S}^{0} x_{j, S t+s}^{* 2}=T^{-2} \sum_{t=1}^{N} \frac{S}{2} X_{t}^{\prime} C_{j}^{*} X_{t}+o_{p}(1) \text { for } \\
& j=1, \ldots, S^{*} \\
& \Rightarrow \frac{1}{2 S} \int_{0}^{1} W(r)^{\prime} \Psi(1)^{\prime} C_{j}^{*} \Psi(1) W(r) d r \\
& =\frac{\sigma^{2}\left(a_{j}^{2}+b_{j}^{2}\right)}{2 S} \int_{0}^{1} W(r)^{\prime} C_{j}^{*} W(r) d r \text { if } j=1, \ldots, S^{*} .
\end{aligned}
$$

Now, we can focus on the denominators and apply the same procedure:

$$
\begin{aligned}
& T^{-2-2 d} \sum_{t=1}^{N} \sum_{S=1-S}^{0} \tilde{x}_{j, S t+S}^{2}=T^{-2-2 d} \sum_{t=1}^{N} S \tilde{X}_{t}^{\prime} C_{j} \tilde{X}_{t}+o_{p}(1) \text { for } \\
& j=0, S / 2 \\
& \Rightarrow \frac{1}{S} \int_{0}^{1} W_{d+1}(r)^{\prime} \Psi(1)^{\prime} C_{j} \Psi(1) W_{d+1}(r) d r
\end{aligned}
$$

$$
=\left\{\begin{array}{l}
\frac{\sigma^{2} \psi(1)^{2}}{S} \int_{0}^{1} W_{d+1}(r)^{\prime} C_{0} W_{d+1}(r) d r \text { if } j=0 \\
\frac{\sigma^{2} \psi(-1)^{2}}{S} \int_{0}^{1} W_{d+1}(r)^{\prime} C_{S / 2} W_{d+1}(r) d r \text { if } j=S / 2 .
\end{array}\right.
$$

The weak convergence of these objects stems from Lemma 2 and the rest can be derived from the above results. Finally, we can also demonstrate,

$$
\begin{aligned}
& T^{-2-2 d} \sum_{t=1}^{N} \sum_{s=1-S}^{0} \tilde{x}_{j, S t+s}^{2}=T^{-2-2 d} \sum_{t=1}^{N} \frac{S}{2} \tilde{X}_{t}^{\prime} C_{j} \tilde{X}_{t}+o_{p}(1) \text { for } \\
& j=1, \ldots, S^{*} \\
& \Rightarrow \frac{1}{2 S} \int_{0}^{1} W_{d+1}(r)^{\prime} \Psi(1)^{\prime} C_{j} \Psi(1) W_{d+1}(r) d r \\
& =\frac{\sigma^{2}\left(a_{j}^{2}+b_{j}^{2}\right)}{2 S} \int_{0}^{1} W_{d+1}(r)^{\prime} C_{j} W_{d+1}(r) d r \text { if } j=1, \ldots, S^{*}
\end{aligned}
$$

$$
\begin{aligned}
& T^{-2-2 d} \sum_{t=1}^{N} \sum_{S=1-S}^{0} \tilde{x}_{j, S t+s}^{* 2}=T^{-2-2 d} \sum_{t=1}^{N} \frac{S}{2} \tilde{X}_{t}^{\prime} C_{j}^{*} \tilde{X}_{t}+o_{p}(1) \text { for } \\
& \quad j=1, \ldots, S^{*} \\
& \Rightarrow \frac{1}{2 S} \int_{0}^{1} W_{d+1}(r)^{\prime} \Psi(1)^{\prime} C_{j} \Psi(1) W_{d+1}(r) d r
\end{aligned}
$$




$$
=\frac{\sigma^{2}\left(a_{j}^{2}+b_{j}^{2}\right)}{2 S} \int_{0}^{1} W_{d+1}(r)^{\prime} C_{j}^{*} W_{d+1}(r) d r \text { if } j=1, \ldots, S^{*}
$$

where the asymptotic objects in (13) and (14) are the same as the arguments made for the numerator counterparts. After obtaining these fundamental objects, for the tests $\tau_{0}(d), \tau_{S / 2}(d), \tau_{j}(d), \tau_{j}^{*}(d)$, $\tilde{\tau}_{j}(d), \tau_{1, \ldots,\lfloor S / 2\rfloor}(d)$ and $\tau_{0, \ldots,\lfloor S / 2\rfloor}(d)$ the results follow by invoking CMT. Furthermore, following BOT, we can write $W(s)^{\prime} C_{j} W(s)=$ $W(s)^{\prime} v_{j} v_{j}^{\prime} W(s)$ for $j=0, S / 2$ and $W(s)^{\prime} C_{i} W(s)=W(s)^{\prime} v_{i} v_{i}^{\prime} W(s)$ for $i=1, \ldots, S^{*}$. We can define $W_{j}(s)=v_{j}^{\prime} W(s)$ for $j=0, S / 2$ and $W_{i}(s)=c_{i}^{\prime} W(s), W_{i}^{*}(s)=c_{i}^{* \prime} W(s)$ for $i=1, \ldots, S^{*}$ as the independent Brownian motions where $c_{i}^{\prime}$ and $c_{i}^{* \prime}$ are the first rows of $v_{i}^{\prime}$ and $v_{i}^{* \prime}$ respectively. Similarly, we can define the corresponding fractional Brownian motions since they are also invariant under any linear transformations.

\section{References}

Barrio Castro, Tomás del, Osborn, Denise R., Taylor, A.M. Robert, 2012. On augmented HEGY tests for seasonal unit roots. Econometric Theory 28 (5), 11211143.
Barrio Castro, Tomás del, Osborn, Denise R., Taylor, A.M. Robert, 2016. The performance of lag selection and detrending methods for HEGY seasonal unit root tests. Econometric Rev. 35 (1), 122-168.

Gregoir, S., 2006. Efficient tests for the presence of a pair of complex conjugate unit roots in real time series. J. Econometrics 130 (1), 45-100.

Hylleberg, Svend, Engle, Robert F., Granger, Clive W.J., Yoo, Byung Sam, 1990. Seasonal integration and cointegration. J. Econometrics 44 (1), 215-238.

Jansson, M., Nielsen, M.Ø., 2011. Nearly efficient likelihood ratio tests for seasonal unit roots. J. Time Ser. Econom. 3 (1).

Marinucci, Domenico, Robinson, Peter M., 2000. Weak convergence of multivariate fractional processes. Stochastic Process. Appl. 86 (1), 103-120.

Nielsen, Morten Ørregaard, 2009. A powerful test of the autoregressive unit root hypothesis based on a tuning parameter free statistic. Econometric Theory 25 (06), 1515-1544.

Nielsen, Morten Ørregaard, 2010. Nonparametric cointegration analysis of fractional systems with unknown integration orders. J. Econometrics 155 (2), 170187.

Robinson, Peter, 2003. Long Memory Time Series. Oxford University Press.

Rodrigues, Paulo M.M., Taylor, A.M. Robert, 2007. Efficient tests of the seasonal unit root hypothesis. J. Econometrics 141 (2), 548-573.

Smith, Richard J., Taylor, A.M. Robert, Barrio Castro, Tomas del, 2009. Regressionbased seasonal unit root tests. Econometric Theory 25 (2), 527-560.

Taylor, A.M. Robert, 2005. Variance ratio tests of the seasonal unit root hypothesis. J. Econometrics 124 (1), 33-54. 